livraisons

d'Histoire

de l'Architecture

\section{Livraisons de l'histoire de l'architecture}

15 | 2008

Chambres de commerces

\title{
La chambre de commerce de Cambrai, vitrine de la reconstruction réussie d'une ville du Nord
}

fr

The Chamber of Commerce and Industry in Cambrai: an example of a successful Reconstruction in a Northern city

Die Industrie- und Handelskammer von Cambrai, Spiegelbild des Wiederaufbaus einer Stadt Nordfrankreichs

\section{Anne Lefebvre et Mathilde Méreau}

\section{OpenEdition}

\section{Journals}

Édition électronique

URL : http://journals.openedition.org/lha/166

DOI : $10.4000 /$ lha. 166

ISSN : 1960-5994

\section{Éditeur}

Association Livraisons d'histoire de l'architecture - LHA

\section{Édition imprimée}

Date de publication : 10 juin 2008

Pagination : 53-66

ISSN : 1627-4970

Référence électronique

Anne Lefebvre et Mathilde Méreau, «La chambre de commerce de Cambrai, vitrine de la reconstruction réussie d'une ville du Nord », Livraisons de l'histoire de l'architecture [En ligne], 15 | 2008, mis en ligne le 10 juin 2010, consulté le 01 mai 2019. URL : http://journals.openedition.org//ha/166 ; DOI : $10.4000 /$ /ha. 166

Ce document a été généré automatiquement le 1 mai 2019.

Tous droits réservés à l'Association LHA 


\section{La chambre de commerce de Cambrai, vitrine de la reconstruction réussie d'une ville du Nord}

fr

The Chamber of Commerce and Industry in Cambrai: an example of a successful Reconstruction in a Northern city

Die Industrie- und Handelskammer von Cambrai, Spiegelbild des Wiederaufbaus einer Stadt Nordfrankreichs

\section{Anne Lefebvre et Mathilde Méreau}

1 Après le rétablissement des chambres de commerce par l'arrêté du 3 nivôse an XI (24 décembre 1802), celle de Lille recommença à fonctionner le 14 février 1803. Sa circonscription s'étendait à l'origine aux arrondissements de Lille, Douai, Cambrai, Valenciennes et Avesnes mais assez rapidement, de nouvelles chambres s'en détachèrent : Valenciennes en 1836, puis Roubaix et Tourcoing en 1872, Cambrai en 1880 et, enfin, en 1887, Armentières et Avesnes. Entre temps, les intérêts économiques du Cambrésis avaient été défendus au sein d'une chambre consultative des arts et manufactures, créée le 19 mars 1848, organisme plus au fait des problèmes locaux, dont les membres se réunissaient à l'hôtel de ville. Le 2 avril 1880, un décret créa la chambre de commerce de Cambrai et les séances continuèrent à se tenir à l'hôtel de ville pendant près de vingt ans. En 1889, le siège fut transféré dans les locaux de la Société des docks et entrepôts, à proximité du port intérieur de Cantimpré, sur l'Escaut, le temps de l'édification d'un hôtel consulaire adossé à des hangars publics ${ }^{1}$. C'était un bâtiment en briques s'apparentant plus à un bâtiment industriel ou à une gare qu'à un bâtiment civil institutionnel; il fut détruit lors des bombardements de 1918.

2 Le Nord fut un des théâtres d'opération les plus importants de la première guerre mondiale. Les attaques allemandes ou alliées furent nombreuses et laissèrent des champs de ruines. Le 26 août 1914, les Allemands entrent dans Cambrai. En novembre 1917, le 
front se déplace d'Ypres vers Cambrai où se déroule la première bataille de chars de l'histoire pour percer la ligne Hindenburg et traverser l'Escaut. Le 7 septembre 1918, la population est évacuée vers Valenciennes et la Belgique, laissant le champ libre aux armées rivales. La ville est alors pillée et incendiée lors de la retraite allemande et une grande partie de l'histoire de la cité disparait dans les flammes. La ville est libérée par les Canadiens le 9 octobre 1918. Du Cambrai médiéval et moderne, ne subsistent que de rares vestiges, à la périphérie ; $20 \%$ des immeubles sont à reconstruire et la Grand-Place n'est plus qu'un lieu de désolation ${ }^{2}$. À la fin de la guerre, la chambre de commerce se trouve sans siège. Elle est accueillie dans les bureaux de la Sucrerie centrale de Cambrai, 15 rue du Louvre à Paris, puis, retourne à Cambrai où l'attend une tâche immense. Son siège migre du 6 place de la Porte Notre-Dame, chez l'un de ses membres, au 10 rue Saint-Géry, dans un immeuble loué et aménagé pour plusieurs années. Se pose alors la question de la reconstruction d'un hôtel consulaire, sur l'ancien emplacement, à l'extérieur de la ville ou au contraire en centre-ville. Cette dernière solution est retenue en 1922 considérant qu'il était opportun de profiter de la Reconstruction pour ériger le nouvel immeuble au cœur de l'agglomération renaissante 3 .

\section{Le plan de reconstruction et sa place dans l'urbanisme de Cambrai}

3 L'ampleur des destructions entraîne, au lendemain de la guerre, de nombreuses réflexions qui aboutissent au vote de la loi Cornudet en avril 1919, imposant, entre autres, des normes de voiries et d'hygiène modernes ${ }^{4}$. Le futur architecte de la chambre, Pierre Leprince-Ringuet (1874-1954)5 s'était intéressé dès 1917 à la question de la reconstruction en participant notamment à une exposition visant à l'établissement de modèles types d'habitations et de fermes pour les régions dévastées, organisée par la société des architectes diplômés par le Gouvernement ${ }^{6}$. À partir de 1915, architectes et urbanistes commencent à penser à l'après-guerre ainsi qu'aux moyens nécessaires à mettre en œuvre en vue d'une reconstruction rapide ${ }^{7}$. Leurs réflexions aboutissent à l'idée alors largement partagée par les contemporains qu'il ne faut pas dénaturer une ville détruite, mais la reconstruire dans l'esprit de ce qui existait pour ne pas perturber davantage les habitants encore traumatisés par le conflit mondial. La ville nouvelle ne devait pas faire oublier l'ancienne, chacun devant pouvoir y retrouver des repères.

L'année 1919 marque un tournant dans la carrière de Leprince-Ringuet puisqu'il participe avec deux de ses confrères, Germain Debré (1890-1948) et Jacques Debat-Ponsan (1882-1942), au concours du plan d'agrandissement et d'embellissement de la ville dont ils sortent lauréats avec le projet « Martin-Martine » ${ }^{8}$. Tous trois étaient passés par l'École des beaux-arts, dans l'atelier de Victor Laloux. Pour le concours de Cambrai, ils dressent un projet moderne ${ }^{9}$ qui séduit le jury par son rationalisme et son esthétique néorégionaliste. Dans les années 1920, Jacques Debat-Ponsan s'occupera en particulier de la reconstruction de la Picardie, région limitrophe, où nombre de bâtiments portent sa marque $^{10}$. Les années d'entre-deux-guerres sont donc pour Pierre Leprince-Ringuet les plus fécondes, au cours desquelles il démontre son talent à Paris et dans le département du Nord, aussi bien dans le domaine de l'architecture civile que religieuse ou funéraire. Son sens du travail et de la perfection l'amène à collaborer avec des artistes locaux ou de renom, toujours avec une grande estime réciproque doublée d'une profonde reconnaissance dont la chambre de commerce de Cambrai est le témoin privilégié. C'est 
précisément dans un souci de respecter l'esprit de la ville et ses apports historiques que Leprince-Ringuet travaille à ce dernier projet, "une construction qui est réellement l'œuvre de l'architecte exprime son esprit en même temps que l'esprit de l'époque ${ }^{11}$. Le projet « Martin-Martine » prend le parti de remodeler le centre-ville autour de l'hôtel de ville en regroupant les services liés à la vie administrative: tribunal, chambre de commerce, poste, perception, sur une nouvelle place à l'arrière de la Grand-Place, et de concentrer l'activité commerciale sur la Grand-Place et dans les rues adjacentes. De nouvelles voies à percer sont ainsi projetées avec des perspectives rectilignes dégageant les grands monuments. Le cahier des charges stipule en outre que l'hôtel de ville doit être reconstruit dans le style du XVIII ${ }^{e}$ siècle et les maisons de la place en style flamand. Ce choix prévaudra pour la construction du nouvel hôtel consulaire. Ce projet de reconstruction et d'embellissement, élaboré par une commission extra communale composée d'architectes, d'ingénieurs, d'entrepreneurs, d'élus locaux et d'artistes est approuvé par le conseil municipal le 27 mars 1920.

5 La mise en place des opérations de reconstruction prit du temps car ce choix d'urbanisme nécessitait de nombreuses expropriations que seul l'État était habilité à mener à bien en rachetant les dommages de guerre. Le 28 mars 1922, une convention signée entre Garin, maire de la ville, et l'État, permet désormais à la commune de se substituer à lui pour l'utilisation des dommages de guerre. Ces problèmes juridiques retardèrent la reconstruction qui ne démarra qu'en 1923 et fut considérée achevée à l'inauguration de l'hôtel de ville en $1932^{12}$.

\section{La mise en place de la nouvelle chambre de commerce}

6 Dès la décision de la reconstruction en centre-ville prise ${ }^{13}$, la chambre de commerce vend l'ancien hôtel consulaire, cherche à acheter des dommages de guerre en centre-ville et confie à l'architecte Ernest Herscher (1870-1939) ${ }^{14}$ une étude préliminaire, estimée à deux millions de francs ${ }^{15}$. L'architecte présente deux projets sommaires sur une surface de 2000 $\mathrm{m}^{2}$, situant le nouvel édifice sur la place à l'arrière de l'hôtel de ville tandis que les membres du bureau de la chambre auraient souhaité une vitrine sur la place d'Armes ${ }^{16}$. Cette solution est abandonnée en novembre $1922^{17}$ et, en compensation, la municipalité consent alors à céder gratuitement une partie du terrain à condition que la chambre accueille dans ses locaux un bureau des P.T.T. En conséquence, le choix du projet est réduit à $1200 \mathrm{~m}^{2}: 874 \mathrm{~m}^{2}$ à acquérir et $330 \mathrm{~m}^{2}$ à fournir par la ville. Le terrain dévolu a la forme d'un $\mathrm{T}$ sur le côté nord de la nouvelle place dénommée alors place administrative. À l'origine, la façade principale d'une longueur de 20 mètres est prévue dans l'axe de l'hôtel de ville. La parcelle était profonde de 30 mètres, les deux branches du T aboutissant pour la partie de gauche, large de 12 mètres, rue de Nice, et sur la partie droite, d'une largeur de 19 mètres 50, rue d'Alger. 53 mètres séparaient les deux rues sur lesquelles les façades du bureau des Postes et de la salle de conférence, allaient se constuire $^{18}$. Il est également spécifié que les futurs immeubles à proximité de la chambre de commerce doivent être frappés d'une servitude d'élévation par rapport à la façade de l'hôtel consulaire pour réaliser un ensemble harmonieux.

7 L'année suivante, le projet prend corps avec la définition des surfaces et une esquisse de budget. Pierre Leprince-Ringuet apparaît dans le procès-verbal de la réunion du bureau du 22 mars 1923 où il est qualifié, avec Ernest Herscher, « d'architecte[s] de la chambre de commerce $»^{19}$. Son rôle dans la définition du plan d'aménagement de la ville, suite au 
concours de 1919, fut probablement la raison de sa collaboration avec Herscher, à moins que des liens personnels n'aient pu les unir. Lors de cette réunion extraordinaire, le conseil d'administration choisit entre plusieurs dessins de la façade soumis par les architectes. Il retient « un projet s'inspirant du style flamand, de ligne sobre, à réaliser en matériaux choisis décoratifs $»^{20}$. Ce nouvel ensemble urbain aura donc une unité de style : un néo-régionalisme teinté d'Art Déco avec des façades de briques et ciment pierré rythmées par des grands pignons. Les travaux sont évalués à 1700000 francs doivent être couverts par les dommages de guerre s'élevant à deux millions.

\section{L'évolution des projets de 1924 à 1930}

Le permis de construire est déposé au début de l'année 1924. Les architectes proposent plans, coupes et élévations datés de janvier, février et mars $1924^{21}$. Le dessin de l'élévation de la façade principale n'est pas conservé mais a été reproduit dans la revue L'Architecture en 1932 (ill. 1) $)^{22}$.

III. 1 : E. Herscher et P. Leprince-Ringuet, élévation principale de la Chambre, Cambrai, janvier 1924

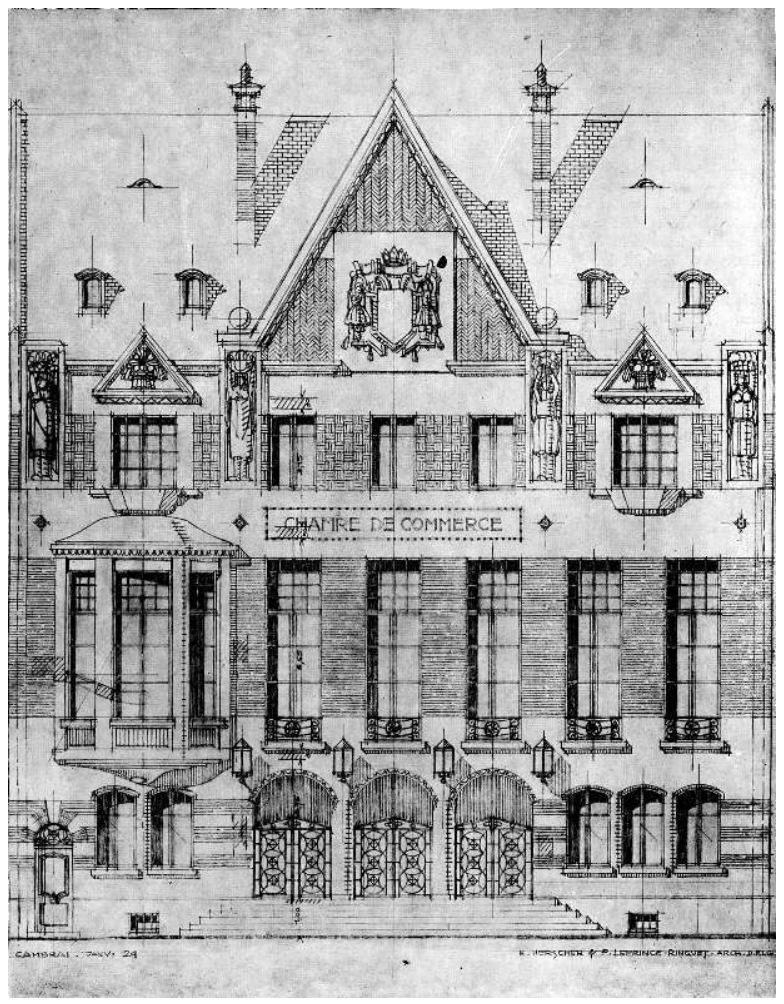

L'Architecture, vol.XLV, n³, 15 mars 1932, p. 76

Cl. C.C.I. de Cambrai

9 Toutefois, les plans de distribution définitifs des trois corps de bâtiments ne sont approuvés qu'en $1928^{23}$. Le projet a peu évolué dans son organisation interne entre les plans de 1924 et ceux effectivement mis à exécution. Aujourd'hui encore, les trois bâtiments formant les trois branches du T prennent jour sur une même cour intérieure d'une surface de $82 \mathrm{~m}^{2}$ (ill. 2). 
III. 2 : E. Herscher et P. Leprince-Ringuet, plan du rez-de-chaussée de la Chambre, Cambrai, s.d. [1924]

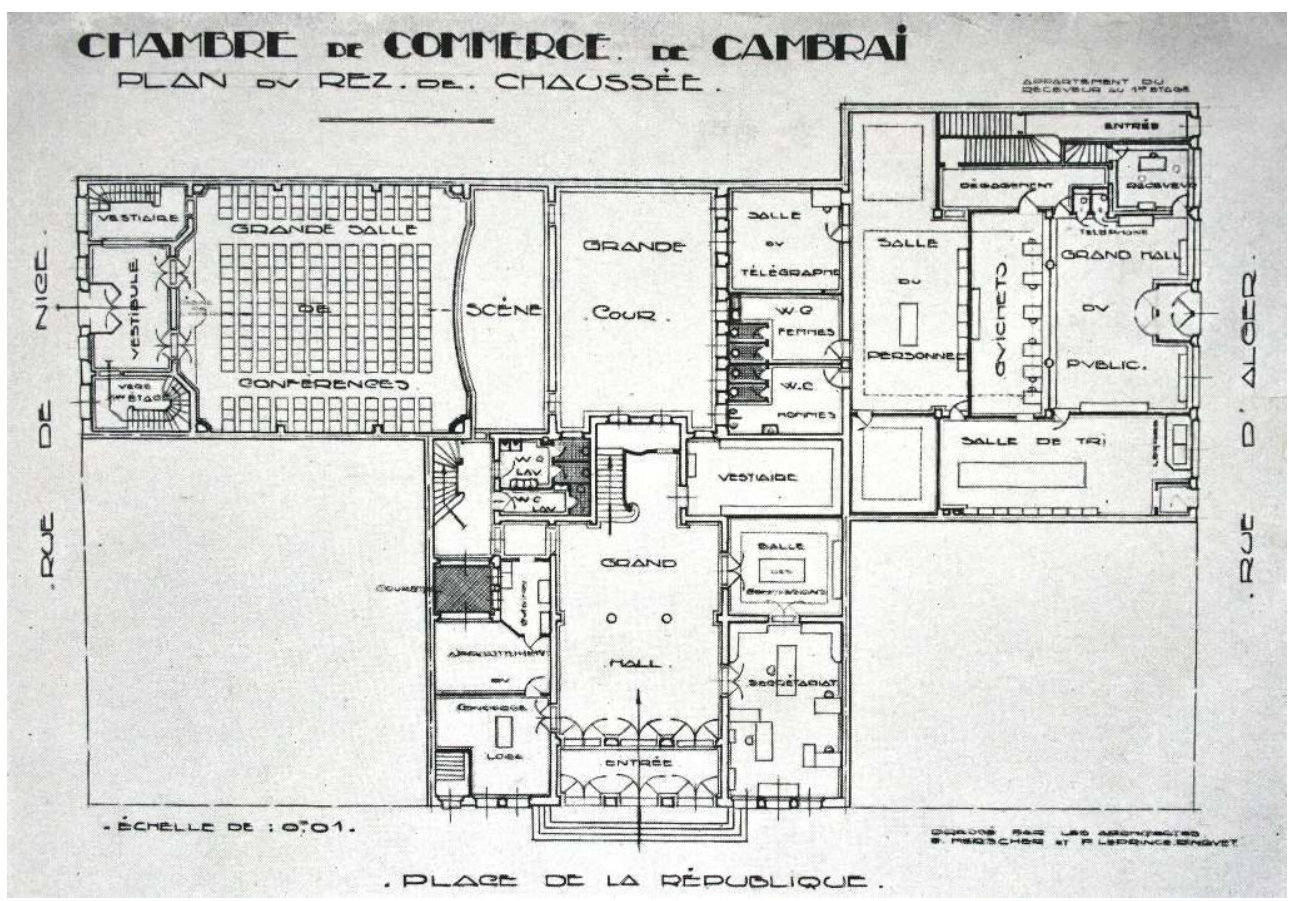

Pierre Leprince-Ringuet, Travaux d'architecture, Strasbourg, s.d. [vers 1934], p. 39

Cl. Olivier Liardet

Le bâtiment principal comprend au rez-de-chaussée un grand hall, la loge du concierge et au premier étage des salles de réunion dont la grande salle des séances ainsi que le bureau du Président. Enfin, au second étage, se situe l'appartement du secrétaire archiviste. Dans le plan de 1924, un ascenseur était prévu à côté de l'escalier d'honneur aboutissant dans un vestibule sur lequel donnaient un vestiaire et une salle de commission. Cet ascenseur disparaît dans la version définitive fusionnant avec le vestibule et le vestiaire pour former un vestibule plus grand et la salle des commissions accueille en même temps sur le mur opposé aux fenêtres une bibliothèque sur deux niveaux avec galerie et escalier.

11 La façade est de rythme ternaire pour la partie centrale, surmontée d'un grand pignon (ill. 3). 


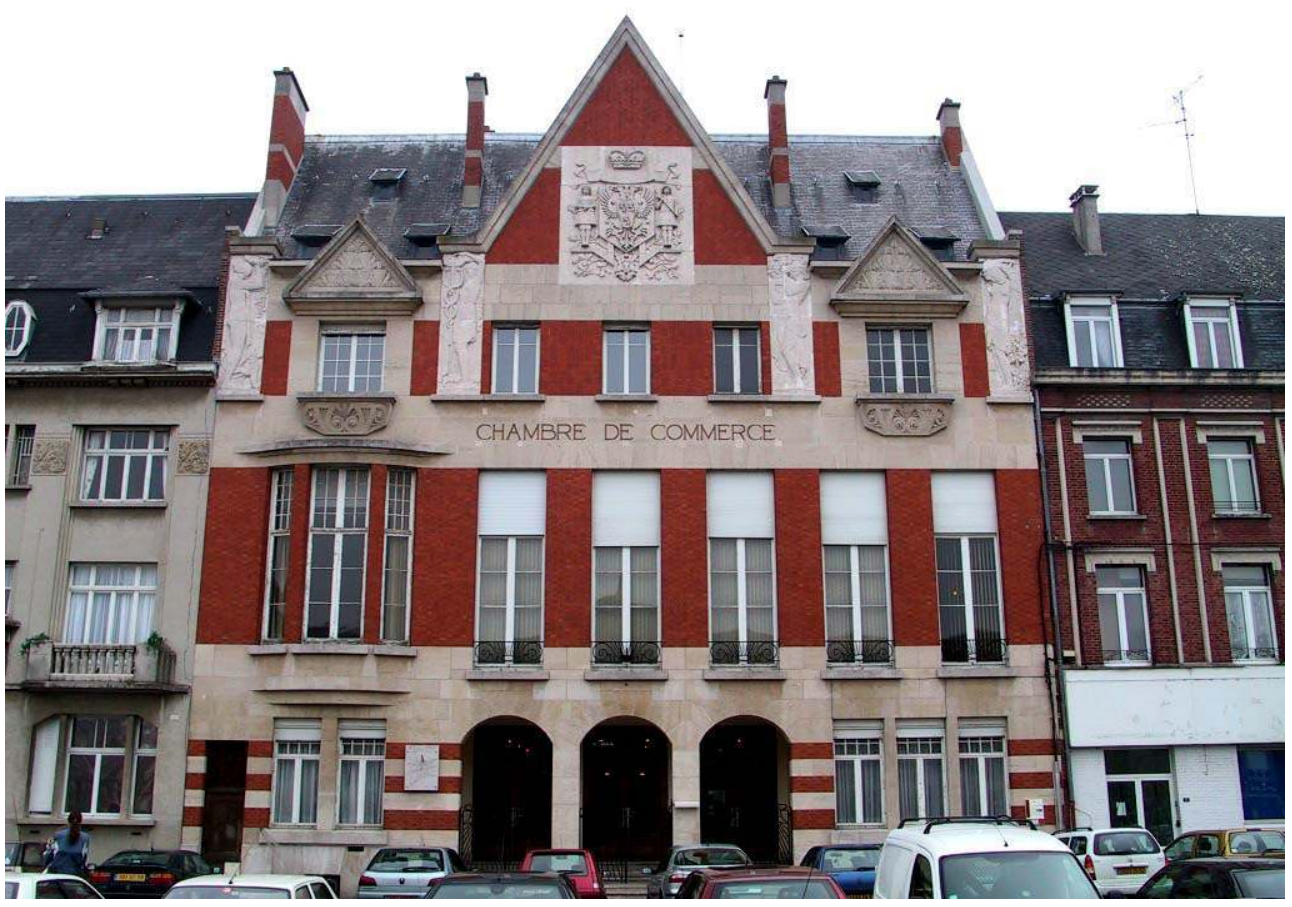

CL. OLIVIER LIARDET

La symétrie n'est assurée qu'au troisième niveau dans les travées latérales doubles où se répondent deux baies s'ouvrant sur un balcon en corbeille et surmontées d'un fronton triangulaire. La verticalité de la travée de gauche est accentuée au premier étage par un bow-window qui éclaire le bureau du président. Au rez-de-chaussée, on accède à l'intérieur par un porche percé de trois arcades. Dans le premier projet, les fenêtres du rez-de-chaussée étaient cintrées, alors que, dans la réalisation définitive, elles ont pris un aspect plus caractéristique de l'Art Déco. Au rez-de-chaussée du bâtiment rue de Nice, une salle de spectacle était envisagée sans communication directe avec le bâtiment de la chambre. Les étages supérieurs étaient cependant intégrés aux espaces de la chambre avec à l'origine, au premier étage la bibliothèque, les archives et le secrétariat, au second, un grand appartement et au troisième, des chambres de service donnant sur la rue et une grande terrasse à l'arrière. La comparaison de l'élévation de 1924 avec l'actuelle permet d'avancer l'hypothèse d'une première façade d'inspiration plus néo-régionaliste par Herscher, composée de cinq travées s'élevant sur deux niveaux plus un niveau de combles avec lucarnes et fronton décoré. Par son style, ce projet était plus proche de la façade principale par son parement de brique, le dessin des ferroneries, des menuiseries et des baies. La façade actuelle n'était pas construite en 1930 et sa réalisation s'éloigne considérablement du parti initial. L'élévation est réduite de moitié probablement pour des raisons de coût, le programme ne comprenant plus qu'une grande salle de conférences avec ses annexes sur la rue, le tout sur deux niveaux. La façade couverte d'un enduit blanc cassé est bien plus sobre, les lignes sont géométriques. Les trois travées centrales rythmées par des pilastres, sont surmontées d'un auvent en béton qui abrite l'entrée et casse la verticalité. Cette dernière version semble plus convenir au style de Leprince-Ringuet et s'apparente à ses constructions contemporaines comme la maison des élèves de l'École centrale ${ }^{24}$. 

classe des P.T.T. et le logement de son receveur (ill. 4).

\section{4 : Façade latérale de la poste rue d'Alger}

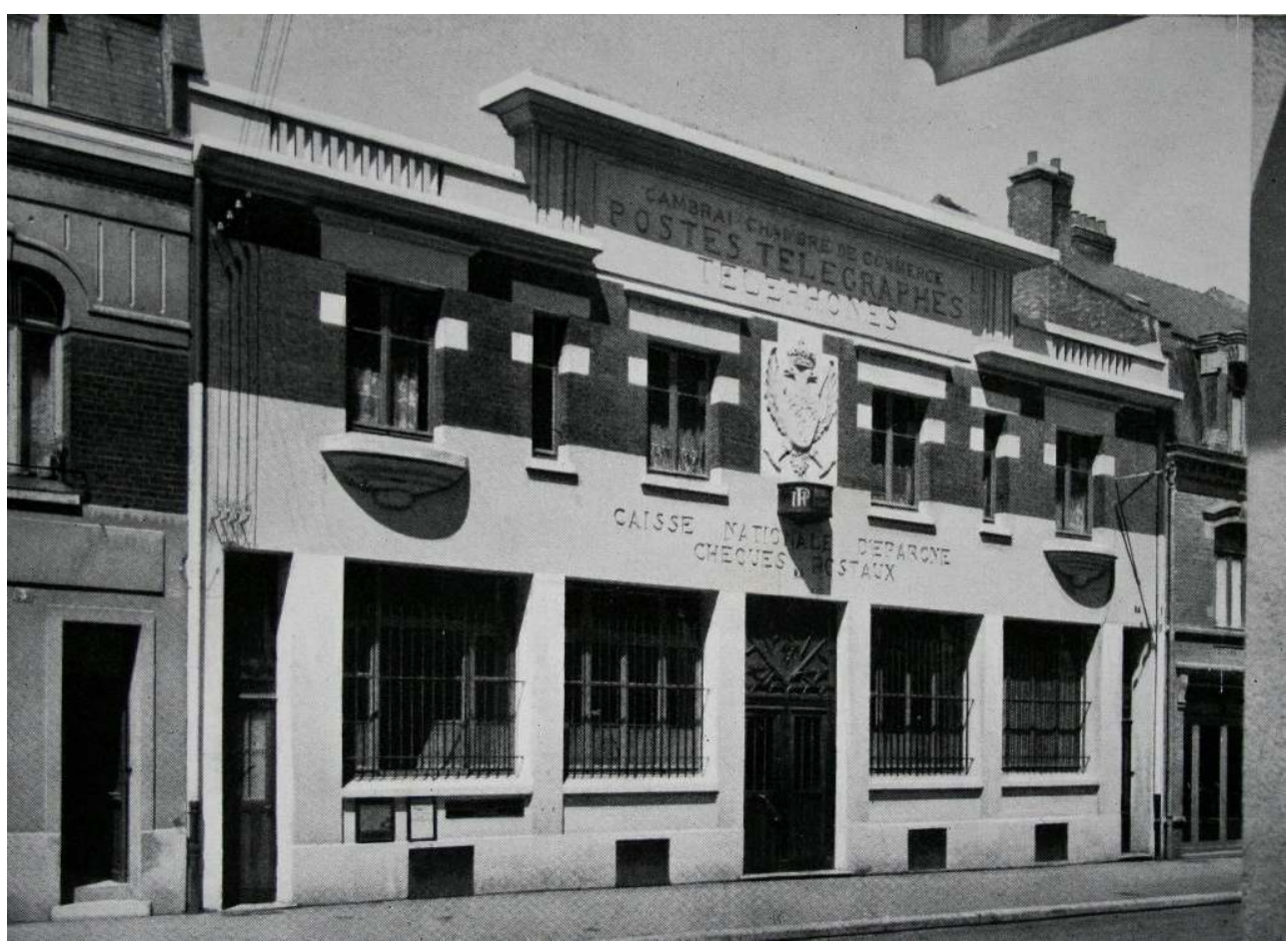

Pierre Leprince-Ringuet, Travaux d'architecture, Strasbourg, s.d. [vers 1934], p. 41

Cl. Olivier Liardet

Elle est créée par arrêté ministériel du 14 août 1923, les plans sont datés de novembre $1924^{25}$. Bien que contemporain, le parti était très différent de la façade sur la rue de Nice, celle-ci étant plus proche des considérations Art Déco. La façade d'origine a été fortement modifiée probablement après la fermeture de la Poste en 1952, tant dans les percements que dans le traitement des matériaux, tout en conservant son allure générale. À l'origine, le rez-de-chaussée était en ciment pierré et l'étage en briques. La façade portait des inscriptions indiquant la destination du bâtiment dans l'entablement surmontant la partie centrale, aujourd'hui disparues. L'élément décoratif était constitué par un écusson aux armes de la ville, axant la façade, au-dessus de la porte d'entrée. Ce bureau des P.T.T. fut ouvert dès le 2 juillet 1928 alors que le reste des bâtiments n'était pas encore terminéz ${ }^{26}$

\section{Le décor de la chambre de commerce de Cambrai}

L'article de la revue l'Architecture paru en 1932 apporte de nombreux renseignements sur la structure et les matériaux du décor conservés jusqu'à nos jours ${ }^{27}$. L'animation de la façade est constituée par le décor sculpté. Une grande sculpture en méplat sur le pignon représente un écusson aux armes de la ville, œuvre du parisien Louis Binet (ill. 5). 
III. 5 : Détail du pignon de la façade principale de la Chambre avec les sculptures de Marcel Gaumont et l'écusson de Louis Binet, juin 2005

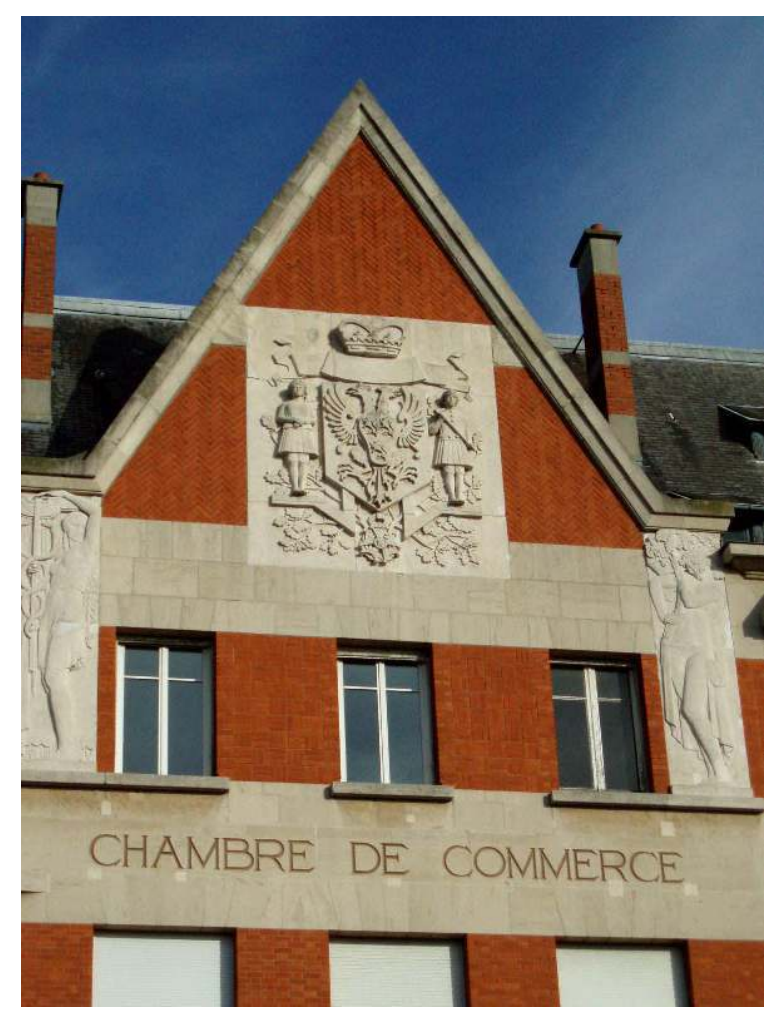

Cl. Anne Lefebvre

16 Au deuxième niveau, les grandes figures en méplat, La Fortune, Le Commerce et L'Abondance sont de Marcel Gaumont (1886-1967) ${ }^{28}$, et les autres parties ornementales de Louis Binet et du Cambrésien Louis Georges. Les ferronneries des garde-corps des fenêtres du premier étage, soulignant la grande salle des séances, ainsi que les grilles fermant les trois arcades du rez-de-chaussée, sont l'œuvre d'un autre artiste Cambrésien, Jean Dubois. On peut remarquer quelques différences entre le projet de 1924 et la réalisation, notamment dans la pente de la toiture où l'on avait prévu des lucarnes à fronton curviligne, remplacées par de simples lucarnes sur le versant. À l'intérieur, le hall, grand espace libre à l'origine, est coupé en son centre par deux colonnes revêtues de mosaïques beiges et dorées qui soutiennent la poutraison maîtresse ; les murs sont plaqués de marbre beige de Lapp jusqu'à hauteur des portes. Le dallage du sol forme un grand motif octogonal central, mélange de marbre, de grès cérame et de granit. L'escalier d'honneur s'ouvre au fond du hall. La rampe, beau travail de ferronnerie de Jean Dubois, est composée de panneaux réguliers dans lesquels alternent nuages et rayons de soleil stylisés, séparés par des montants entre lesquels s'inscrit le caducée entrelacé de serpents, attribut du commerce. À mi-hauteur, un grand vitrail à deux travées représente, dans une perspective volontairement conventionnelle, tous les grands monuments du Cambrai historique ; en avant-plan, les portes de la ville et la maison espagnole; alignés au centre de la composition, les trois clochers, Saint Géry, Saint Martin et la cathédrale. Les différents plans sont très imbriqués, les toitures jouant un rôle de liaison. À l'arrière-plan, les usines et leurs cheminées se fondent dans les champs où l'on voit le soleil se coucher à gauche et se lever à droite. L'ensemble est traité en camaïeu. Les blancs sont obtenus par la variété des qualités de verre: dépolis, striés, cannelés, martelés avec des effets de gravures 
obtenus à l'acide et au jet de sable. Les blancs sont soutenus par des aplats d'un noir vert. Les bordures et les guirlandes sont dans un ton jaune paille. Les verres industriels sont dépolis à l'acide fluorhydrique permettant de créer un dessin ou de contraster et de donner des effets différents. Ce procédé, originalité du maître-verrier Auguste Labouret (1871-1964) ${ }^{29}$ à Cambrai, n'a pas eu de suite à cause de la difficulté de mise en œuvre du polissage à l'acide.

17 La galerie du premier étage, vaste palier, dessert face à l'escalier, la grande salle des séances et à l'extrémité sur la gauche, une salle de réunion (à l'origine grand vestiaire) donnant accès à la salle des commissions-bibliothèque. Deux verrières éclairent cette galerie, prenant le jour sur les cours intérieures, se répondant face à face. Elles ont pour programme le modernisme citadin et rural. À droite, ce sont de hautes cheminées d'usines, dont les fumées se fondent aux nuages. Les toits coniques des malteries s'entrecroisent avec d'autres silhouettes de réservoirs et de bâtiments industriels. À gauche, des éléments de matériel empruntés aux sucreries encadrent un paysage où canaux, ponts et voies ferrées se superposent à un premier plan de meules et d'instruments agricoles (ill. 6).

III. 6 : Galerie du $1^{\text {er }}$ étage, vitrail de gauche par Auguste Labouret figurant des éléments de matériel de sucreries encadrant un paysage de canaux, ponts et voies ferrées et au premier plan de meules et d'instruments agricoles, septembre 2007

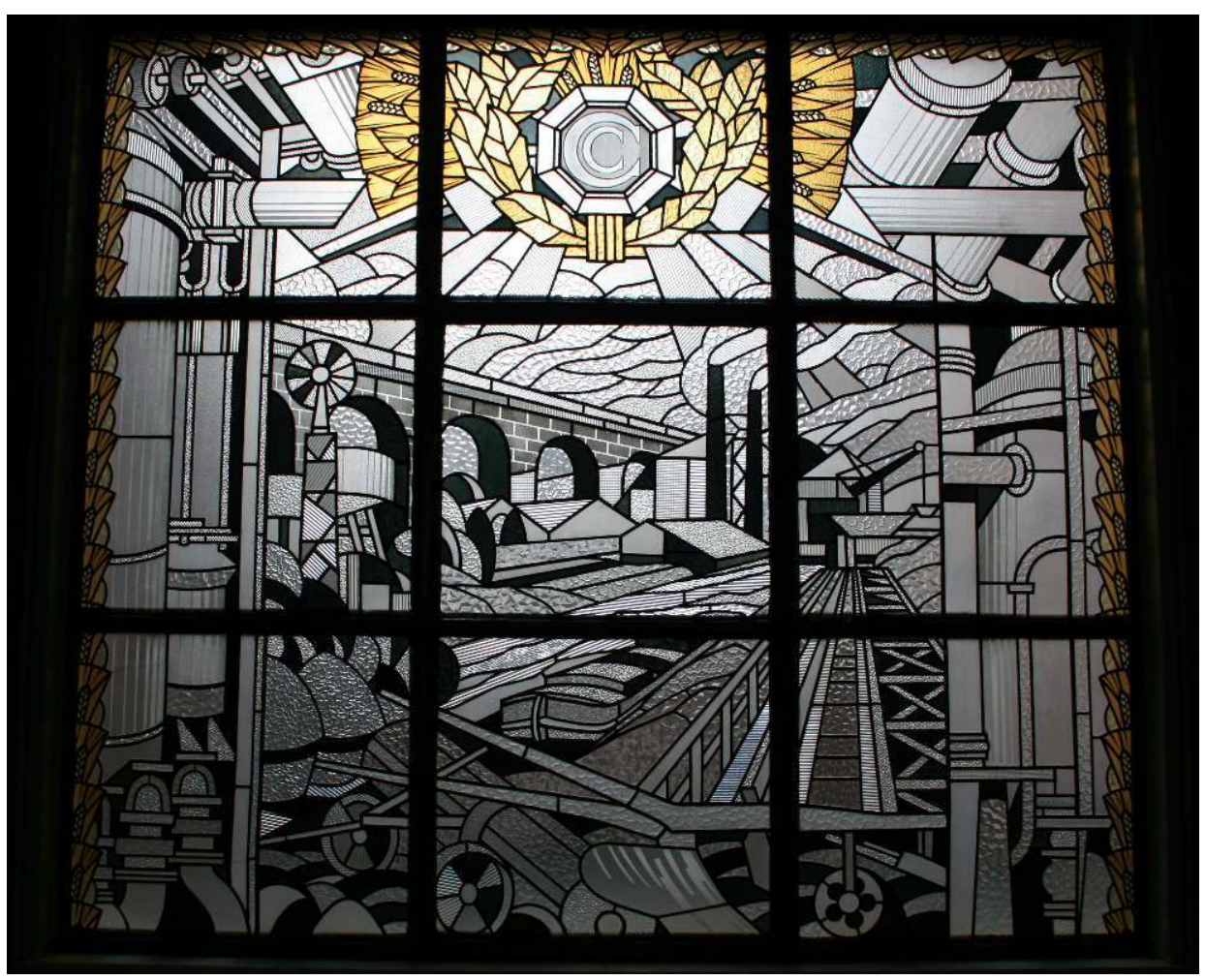

CL. OLIVIER LIARDET

18 Le cartouche central lie la feuille de betterave à l'épi de blé. Avec le vitrail de l'escalier, cet ensemble de trois verrières est un remarquable exemple de l'œuvre de Labouret $^{30}$. La grande salle des séances ouvre par cinq baies sur la place (ill. 7). 
III. 7 : Salle des séances, vue générale vers la cheminée surmontée d'un panneau de Gustave Jaulmes et montrant le mobilier dessiné par Leprince-Ringuet, septembre 2007

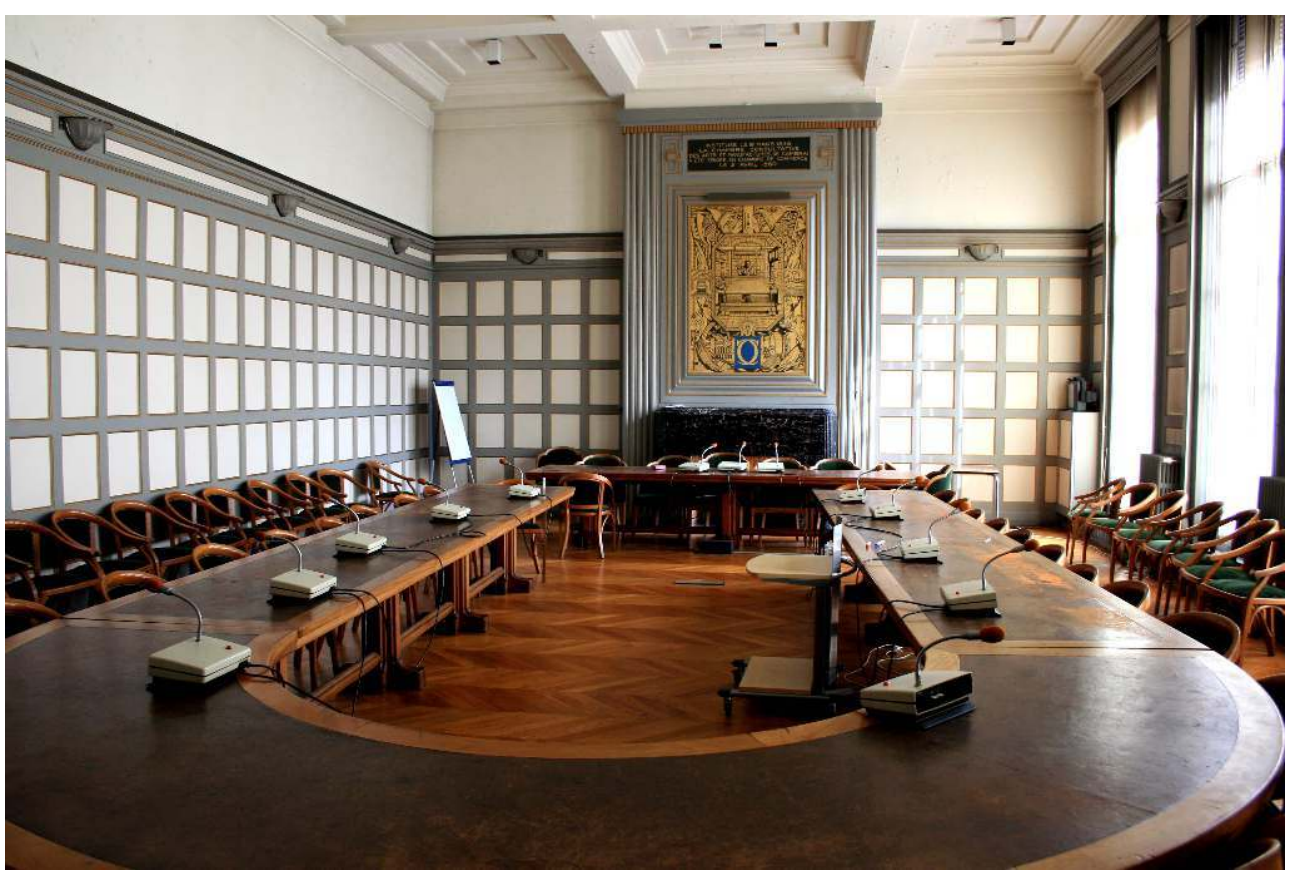

CL. OLIVIER LIARDET

19 Les murs sont couverts de lambris, formés de compartiments réguliers peints d'un ton gris vert, et les poutres du plafond sont apparentes. Sur le mur du fond, à gauche, surmontant une imposante cheminée de marbre, Gustave Jaulmes (1873-1959) ${ }^{31}$ a peint un vieux tisserand installé à son métier, entouré des attributs du commerce et de l'industrie, sur un fond d'or. Lui faisant face, une grande carte du Cambrésis a été exécutée par Émile Flamant (1896-1975) $)^{32}$ sur un carton de Leprince-Ringuet. Au-dessus d'une vue panoramique de Cambrai, toutes les localités de l'arrondissement sont représentées avec les bâtiments qui symbolisent l'activité économique (cheminée de manufacture, moulin, brasseries, métiers à dentelles), leurs églises et leurs blasons. Une grande table en fer à cheval et les fauteuils assortis meublent cette pièce. Le bureau du président, qui a conservé son mobilier d'origine, communique avec la salle des séances. Dans la bibliothèque, ancienne salle des commissions, un grand meuble en chêne clair garnit entièrement le plus long côté de la pièce. À l'extrémité, un escalier en colimaçon donne accès à la galerie supérieure, conçue pour le passage d'une personne. Une cheminée en marbre occupe le mur du fond. Le mobilier et les luminaires, dessinés en grande partie par Pierre Leprince-Ringuet, sont restés en place $^{33}$.

\section{Entre néo-régionalisme et Art Déco}

Le style de la chambre de commerce répond à la figure imposée par le conseil d'administration, c'est-à-dire le style flamand. Mais un tel parti se révèle contradictoire si l'on tient compte que le style flamand s'entend par son opulence, tant par la ligne, courbes et contre-courbes, que par le vocabulaire architectural (baies à meneaux, pignons à redents, lucarnes) et les effets décoratifs, notamment dans les jeux de mise en œuvre de la brique et dans les décors sculptés. On sent à Cambrai une certaine retenue des 
architectes qui s'inscrivent dans la veine néo-régionaliste, illustrée magnifiquement à la chambre de commerce de Lille de Louis-Marie Cordonnier (1854-1940). Les lignes de l'hôtel consulaire de Cambrai se révèlent sensiblement plus tempérées que celles que Jacques Alleman (1882-1945) applique à la reconstruction de Béthune ${ }^{34}$ où celui-ci franchit les limites du style néo-régionaliste dans sa version Art Déco privilégiant des formes géométriques. À Cambrai, l'interprétation du style flamand reste modérée même si, localement, le néo-régionalisme perdure tardivement ${ }^{35}$. Elle se lit certes sur le bâtiment, mais se révèle surtout dans l'ensemble urbain que la chambre de commerce forme en harmonie avec les autres bâtiments de la place. Refusant un caractère ostentatoire, l'institution porte les espoirs du renouveau économique du Cambrésis en reflètant l'état d'esprit d'hommes pragmatiques qui souhaitaient un bâtiment essentiellement fonctionnel, au delà des seules exigences esthétiques. Au cours de l'inauguration, le 16 juin 1930, présidée par le ministre du commerce et de l'industrie, Flandrin, la réalisation fut saluée par un concert de louanges adressées aux architectes comme aux artistes présents ${ }^{36}$. Vitrine du commerce et de l'industrie cambrésienne, la nouvelle chambre de commerce constitue aujourd'hui un témoignage majeur de l'architecture publique de la première reconstruction dans le Nord, qui vient d'être consacré par le label » patrimoine du XXe siècle » attribué par le ministère de la culture et de la communication.

\section{NOTES}

1. 1. Sur l'histoire de la création de la chambre de commerce de Cambrai, voir C comme centenaire. Histoires d'entreprises centenaires de la région Nord-Pas-de-Calais, Lille, 1992, $169 \mathrm{p}$

2. 2. Robert Vandenbussche, "Cambrai dans les drames nationaux, 1914-1918", dans Louis Trénard (dir.), Histoire de Cambrai, Lille, Presses Universitaires de Lille, 1982, p. 259-270.

3. 3. Cambrai, archives de la chambre de commerce et d'industrie (abrégé en Arch. de la CCI), procès-verbal de séance du 15 avril 1922.

4. 4. Loi du 14 mars 1919 sur les plans d'aménagement, d'embellissement et d'extension des villes de plus de 10000 habitants.

5. 5. Grand prix de Rome en 1904, il était également centralien et ses réalisations témoignent d'une alliance de la raison et de la technique, de l'art et du fonctionnel. À Paris, il construit pour les étudiants des résidences ancrées dans l'esprit des années 1930 : maisons des élèves de l'École centrale et de l'École des mines. Villas en bord de mer ou à la campagne lui sont également commandées tout comme, fruit de sa riche collaboration avec le sculpteur Marcel Gaumont (1888-1962), de nombreux monuments aux morts. Une part importante de sa carrière se déroule à Cambrai et dans le Cambrésis. Voir Pierre Leprince-Ringuet, Travaux d'architecture, Strasbourg, Edari, s.d. [vers 1934] ; Mathilde Méreau, Pierre Leprince-Ringuet (1874-1954), formation et carrière, mémoire de master 1, sous la direction de Barthélémy Jobert et Simon Texier, Université Paris IV-Sorbonne, juin 2007 ; et du même auteur, Pierre Leprince-Ringuet (1874-1954), architecte, mémoire de master 2, sous la direction de Barthélémy Jobert, université Paris IV-Sorbonne, [en cours]. 
6. 6. Pierre Leprince-Ringuet, «Fermes de moyenne culture ", dans Fermes et habitations rurales. Projets primés au concours ouverts entre les architectes français, troisième région: MeuseVosges, Meurthe et Moselle, Alsace, Paris, 1917, 3 vol., 100 pl.

7. 7. Reconstructions en Picardie après 1918, cat. exp., Archives départementales de l'Aisne et al., Paris, Réunion des musées nationaux, 2000, $311 \mathrm{p}$.

8. 8. Du nom des deux jacquemarts du carillon de l'hôtel de ville commémorant l'exploit de deux géants, forgerons de leur métier qui, vers 1370 , se trouvaient au nombre des bourgeois partis combattre le seigneur de Thun-Lévêque, accusé de rançonner et de désoler le Cambrésis. Il convient de dire ici que les archives municipales de Cambrai sont inaccessibles dans leur grande majorité notamment pour les travaux d'urbanisme et d'architecture à l'exception de certains plans issus des permis de construire. Celles de la Chambre de commerce ne sont pas classées, seuls sont disponibles certains registres de procès-verbaux de l'assemblée générale et du bureau.

9. 9. Paris, Institut français d'architecture. 013 Ifa 110/2, « Projet de voirie nouvelle pour la ville de Cambrai ", devis descriptif du projet non signé, s.d.

10. 10. Direction régionale des affaires culturelles Picardie, Architectures de la Reconstruction dans l'Est de la Somme, Lyon, Éditions Lieux Dits, 2006, 64 p.

11. 11. Pierre Leprince-Ringuet, Travaux d'architecture, Strasbourg, Éditions Edari, s.d. [vers 1934], $90 \mathrm{p}$.

12. 12. Jean-François Machon, Reconstruction et urbanisme à Cambrai dans l'entre-deux guerres, D.E.A. histoire et civilisation, sous la direction de Bernard Ménager, université de Lille III, 1994, 313 p.

13. 13. Cambrai, arch. de la CCI, procès-verbal de séance du 15 avril 1922.

14. 14. Architecte à Paris, membre de la société des architectes diplômés par le gouvernement et ancien de l'atelier de Chancel et Jean-Louis Pascal à l'École des beaux-arts, promotion de 1891, diplômé le 17 juin 1898. Son intervention à la Chambre est probablement le fruit d'une entremise de son frère, René Herscher, blanchisseur à Cambrai, membre du conseil municipal et de la chambre de commerce et président du comité d'arrondissement de reconstitution industrielle.

15. 15. Cambrai, arch. de la CCI, procès-verbal de séance du 29 mai 1922.

16. 16. Cambrai, arch. de la CCI, procès-verbal de séance du 3 juillet 1922.

17. 17. Cambrai, arch. de la CCI, procès-verbal de séance du 7 novembre 1922.

18. 18. Cambrai, arch. de la CCI, procès-verbal de la réunion extraordinaire du 22 mars 1923.

19. 19. Ibid.

20. 20. Ibid.

21. 21. Seuls les plans ont pu être retrouvés aux archives municipales, les pièces écrites demeurent à ce jour introuvables.

22. 22. Félix Ollivier, «La chambre de commerce de Cambrai », L’Architecture, vol. XLV, $\mathrm{n}^{\circ} 3,15$ mars 1932, p. 76.

23. 23. Cambrai, arch. de la CCI, procès-verbal de séance du 27 mai 1928. Les plans non annexés au procès-verbal n'ont pas été retrouvés.

24. 24. Maison des élèves de l'École centrale, 30 boulevard Diderot, Paris $\left(11^{\mathrm{e}}\right)$.

25. 25. Lille, arch. dép. du Nord, 0121/709, arch. mun. de Cambrai, élévation de la façade rue d'Alger.

26. 26. Le Cambrésis renaît de ses cendres, Cambrai, 1930, p. 154-156.

27. 27. Félix Ollivier, «La chambre de commerce de Cambrai ", L'Architecture, vol. XLV, $\mathrm{n}^{\circ}$ 3, 15 mars 1932, p. 73-84.

28. 28. Maryvonne Lencot, Redécouverte de l'œuvre sculptée de Marcel Gaumont (1880-1962), mémoire de maîtrise d'histoire de l'art sous la direction de Bruno Foucart, université Paris IV-Sorbonne, 1988. Grand prix de Rome de sculpture en 1908, Gaumont est membre de l'Institut à partir de 1945. 
29. 28. Ginette Day, «Un artiste picard de renommée internationale: Auguste Labouret (1871-1954), maître-verrier, maître mosaïste », Hirson, musée centre de documentation Alfred Desmasures, 1995, non paginé.

30. 30. Inscription au titre des monuments historiques de la cage d'escalier, de la rampe d'escalier et de l'ensemble des vitraux par arrêté préfectoral du 30 janvier 2006.

31. 31. Grand Prix de Rome, il a décoré le théâtre de Carcassonne, le grand foyer du palais de Chaillot et celui du bureau du travail international à Genève.

32. 32. Peintre fresquiste, il a décoré la salle des mariages de l'hôtel de ville de Cambrai et peint de grands décors religieux dans les églises de la reconstruction du Cambrésis.

33. 33. À l'exception des grands lustres de la salle des séances qui se trouvent à la mairie d'Iwuy (Nord).

34. 34. Émilie Piquavet, La Reconstruction de Béthune après la première guerre mondiale, maîtrise d'histoire, sous la direction d'Eric Buissière, université d'Artois, 1997-1998, 340 p.

35. 35. Sur l'histoire du mouvement néo-régionaliste voir Benoît Mihail, Une Flandre à la française, l'identité régionale à l'épreuve du modèle républicain, Paris, Éditions Labor, 2006, 422 p.

36. 36. « Compte-rendu de la cérémonie du cinquantenaire de la fondation de la chambre de commerce de Cambrai et de l'inauguration de son hôtel, place de la République à Cambrai », Bulletin mensuel de la chambre de commerce de Cambrai, 15 juin 1930, p. 15.

\section{RÉSUMÉS}

Les destructions de la Grande Guerre laissent la ville de Cambrai en ruines. En 1919, Pierre Leprince-Ringuet, Jacques Debat-Ponsan et Germain Debré remportent le concours pour le plan d'agrandissement et d'embellissement de Cambrai. Architecte de la reconstruction, Pierre Leprince-Ringuet veillera à conserver l'esprit de la ville ancienne, refondant les artères et créant de nouveaux espaces urbains en utilisant la tradition architecturale flamande. Avec René Herscher, il réalise la nouvelle chambre de commerce située sur une place à l'arrière de l'hôtel de ville. Le programme intègre en outre un bureau de poste et une salle de conférences. La complexité du règlement des dommages de guerre retarde le démarrage des travaux jusqu'en 1923. Les architectes font appel à des artistes renommés, Marcel Gaumont pour la sculpture et Auguste Labouret pour les vitraux et ces interventions contribueront fortement à imprimer un caractère Art Déco mais également néo-régionaliste au palais consulaire cambrésien inauguré en 1930.

Destruction caused by the Great War had left the city of Cambrai in ruins. In 1919, Pierre Leprince-Ringuet, Jacques Debat-Ponsan and Germain Debré won the competition for the plan for the extension and improvements of Cambrai. As architect of the Reconstruction, Pierre LeprinceRinguet saw to it that the spirit of the old city was preserved: he built the roads on new foundations and created new urban spaces while using Flemish architectural tradition. In collaboration with René Herscher, he achieved the new Chamber of Commerce located on a place behind the town hall. The program also included a post office and a lecture room. The construction work was delayed to 1923, owing to the complex settlement of war damages. The architects called on some well-known artists such as Marcel Gaumont for sculpture and Auguste 
Labouret for stained-glass windows: these works will definitely give an Art Deco but also NeoRegionalism character to the consular palace of Cambrai inaugurated in 1930.

Nach den Zerstörungen des ersten Weltkrieges lag die Stadt Cambrai in Trümmern. 1919 gewannen Pierre Leprince-Ringuet, Jacques Debat-Ponsan und Germain Debré den Wettbewerb für die Planung der Erweiterung und der Verschönerung Cambrais. Pierre Leprince-Ringuet, Architekt des Wiederaufbaus, sorgte dafür, den Geist der früheren Stadt zu bewahren, indem er Straßen neu fasste und neue Stadträume schuf, die die flämische architektonische Tradition übernahmen. Gemeinsam mit René Herscher erstellte er die neue Handelskammer auf einem Platz hinter dem Rathaus. Das Programm beinhaltete außerdem ein Postamt und einen Konferenzraum. Die Umständlichkeit der Regelung von Kriegsschäden verzögerte das Starten der Bauarbeiten bis 1923. Die Architekten beriefen dazu renommierte Künstler, den Bildhauer Marcel Gaumont und den Glasmaler Auguste Labouret. Deren Beiträge verliehen dem konsularischen Palast von Calais, 1930 eingeweiht, ein starkes Art-Deco-Gepräge, ebenso einen neoregionalistischen Charakter.

\section{AUTEUR}

\section{ANNE LEFEBVRE ET MATHILDE MÉREAU}

Anne Lefebvre est titulaire d'une licence d'histoire de l'art et d'une licence d'histoire et de géographie (Lille 3). Elle est chargée d'études documentaires à la DRAC Nord/Pas-de-Calais et s'occupe de la protection des monuments historiques depuis 1978. Membre de la commission supérieure des monuments historiques, section patrimoine industriel, scientifique et technique (jusqu'en 2007), membre de la commission historique du Nord et de la commission départementale d'histoire et d'archéologie du Pas-de-Calais, elle a notamment publié un article consacré à « La chapelle Sainte-Thérèse et de la Sainte-Face à Hem » dans le Bulletin de la commission historique du Nord (t. XLIX, 1999) et a participé à la rédaction des Itinéraires du patrimoine sur le patrimoine minier : Le Bassin minier entre Scarpe et Escaut et De Lens à Auchel (Itinéraire $n^{\circ} 89$ et 90, Lille, 1995) et Roubaix, une ville née de l'Industrie (Itinéraire $n^{\circ} 205$, Lille, 2000). Anne Lefebvre vient de rédiger les notices sur les jardins du Nord/Pas-de-Calais pour Le Guide des Jardins de France, dirigé par Michel Racine aux Éditions Ulmer publié en 2007 et des notices sur l'architecture roubaisienne à paraître prochainement dans le guide Monum consacré à la ville de Roubaix. Adresse électronique : anne.lefebvre@culture.gouv.fr Mathilde Méreau est licenciée d'histoire de l'art (Lille 3, 2006). Après avoir travaillé sur la formation et la carrière de l'architecte Pierre Leprince-Ringuet pour son mémoire de master 1 , soutenu en juin 2007, sous la direction de Barthélemy Jobert et de Simon Texier (Paris IVSorbonne), elle poursuit ses recherches en s'intéressant aux réalisations parisiennes de Pierre Leprince-Ringuet et son travail autour de la reconstruction de Cambrai pour son mémoire de master 2. Adresse électronique : mathilde.mereau@neuf.fr 